The following text is now added to the beginning of the fourth paragraph of the 'Statistical analysis' subsection of the Methods section: "A normalization factor can be added to account for the uncertainty in the symptom onset dates of the index cases. Assuming a uniform distribution, the likelihood would only differ by a multiplicative constant and give the same estimates." Also, the time frame in the final sentence of that paragraph ("from day 1 to 7") was incorrect. The correct time frame is "from days 5, 8 and 11."

Finally, Fig. 1c and Extended Data Fig. 1 have been replaced accordingly, and the legend to Extended Data Fig. 1 ("to start from 1 days (top left) to 7 days (bottom right) before symptom onset") is now rephrased ("to start from 5 days (top), 8 days (middle) and 11 days (bottom) before symptom onset") to match the revised figure.

The errors have been corrected in the HTML and PDF versions of the article.

The original code has also been replaced with updated code in Supplementary Software 1.

Published online: 7 August 2020

https://doi.org/10.1038/s41591-020-1016-Z

(c) The Author(s), under exclusive licence to Springer Nature America, Inc. 2020

\title{
Author Correction: Sampling the host response to SARS-CoV-2 in hospitals under siege
}

Alexander W. Charney, Nicole W. Simons, Konstantinos Mouskas, Lauren Lepow, Esther Cheng, Jessica Le Berichel, Christie Chang (1D, Robert Marvin, Diane Marie Del Valle, Sharlene Calorossi, Alona Lansky, Laura Walker, Manishkumar Patel, Hui Xie, Nancy Yi, Alex Yu, Gurpawan Kang @D, Lora E. Liharska, Emily Moya, Matthew Hartnett, Sandra Hatem, Lillian Wilkins, Melody Eaton, Hajra Jamal, Kevin Tuballes, Steven T. Chen, Jonathan Chung, Jocelyn Harris, Craig Batchelor, Jose Lacunza, Mahlet Yishak, Kimberly Argueta, Neha Karekar, Brian Lee, Geoffrey Kelly, Daniel Geanon, Diana Handler, John Leech, Hiyab Stefanos, Travis Dawson, leisha Scott, Nancy Francoeur, Jessica S. Johnson, Akhil Vaid, Benjamin S. Glicksberg DD, Girish N. Nadkarni, Eric E. Schadt, Bruce D. Gelb, Adeeb Rahman, Robert Sebra, Glenn Martin, The Mount Sinai COVID-19 Biobank Team, Thomas Marron, Noam Beckmann, Seunghee Kim-Schulze, Sacha Gnjatic (1) and Miriam Merad

Correction to: Nature Medicine https://doi.org/10.1038/s41591-020-1004-3, published online 27 July 2020.

In the version of this article initially published, an author name in the Mount Sinai COVID-19 Biobank Team list ('Gavin Grimes') was incorrect. The correct name is 'Gavin Gyimesi. The error has been corrected in the HTML and PDF versions of the article.

${ }^{\star} \mathrm{A}$ list of authors and their affiliations appears online.

Published online: 21 August 2020

https://doi.org/10.1038/s41591-020-1057-3

(c) The Author(s), under exclusive licence to Springer Nature America, Inc. 2020 